\title{
Sticking points in magnetic resonance diagnosis of constrictive pericarditis
}

\author{
Donald D. Glower, MD
}

\author{
From the Department of Surgery, Duke University Medical Center, Durham, NC. \\ Disclosures: Author has nothing to disclose with regard to commercial support. \\ Received for publication Dec 27, 2015; accepted for publication Dec 30, 2015. \\ Address for reprints: Donald D. Glower, MD, Department of Surgery, Duke University Medical Center, Box \\ 3851, Room 4554 Blue Zone, Durham, NC 27710 (E-mail: glowe001@mc.duke.edu or d.glower@dm. \\ duke.edu). \\ J Thorac Cardiovasc Surg 2016;151:1356-7 \\ $0022-5223 / \$ 36.00$ \\ Copyright (c) 2016 by The American Association for Thoracic Surgery \\ http://dx.doi.org/10.1016/j.jtcvs.2015.12.042
}

In their article in this issue of the Journal, Power and colleagues ${ }^{1}$ present data regarding the use of cardiac magnetic resonance $(\mathrm{CMR})$ radiofrequency tagging to diagnose constrictive pericarditis (Figure 1). Power and colleagues ${ }^{1}$ conclude that $\mathrm{CMR}$ with radiofrequency tissue tagging offers a unique, efficient, and effective method of defining clinically and surgically relevant constrictive pericarditis and that catheterization has substantial and unacceptable false-positive and false-negative rates, with important clinical ramifications.

Although CMR with radiofrequency tagging does seem to detect the sticking of epicardium to the pericardium, there remain several sticking points in diagnosing constrictive pericarditis by means of CMR with radiofrequency tagging. First, none of the patients in this study had previous cardiac surgery, in which pericardial adhesion is nearly universal to some degree, but constrictive pericarditis is in fact rare. In most Western series, roughly half of the patients with constrictive pericarditis had previous cardiac surgery, although tuberculous pericarditis remains a predominant cause of constrictive pericarditis worldwide. It is of both theoretic and practical concern that this technique only detects pericardial adhesions. Pericardial constriction accounts for a small subset of the many patients with pericardial adhesions. Data are needed to see to what extent CMR can differentiate pericardial adhesions without constriction from true pericardial constriction in a population with known or likely pericardial adhesions, such as those with previous cardiac surgery. In theory, CMR could miss constrictive pericarditis if there were no adhesions between the pericardium and epicardium in a rare but known form of "effusive constrictive pericarditis."

In this study, Power and colleagues ${ }^{1}$ chose to look solely at tissue tagging to diagnose constrictive pericarditis from pericardial adhesions and chose to not use CMR to detect other findings such as septal thickening, septal bounce, dilated venae cavae, interventricular dependence, and altered respiratory variation of mitral or

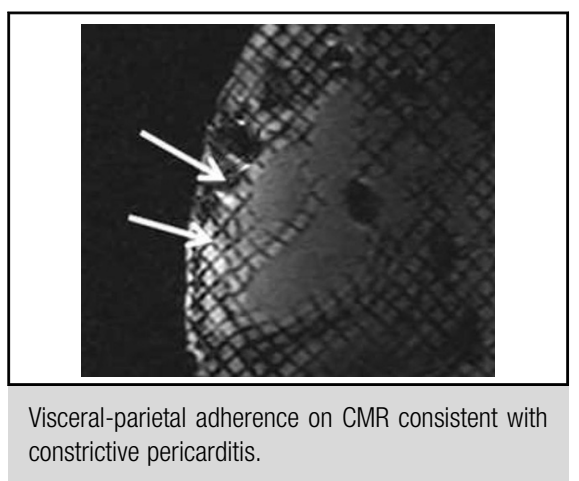

\section{Central Message}

Magnetic resonance may have a role in diagnosing constrictive pericarditis but needs validation in a broader clinical population.

See Article page 1348.

tricuspid inflow patterns. It is very possible that in populations such as those with previous cardiac surgery, use of CMR to examine some of these additional correlates of constrictive pericarditis may be necessary to improve the specificity of CMR in the diagnosis of pericardial constriction. It certainly is not true that all patients with pericardial adhesion according to CMR have constrictive

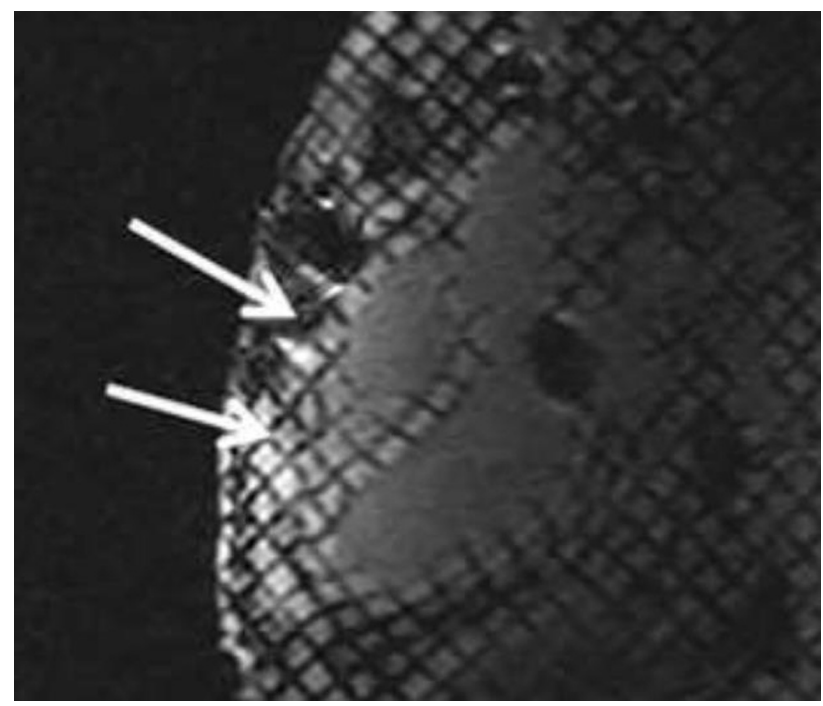

FIGURE 1. Visceral-parietal adherence on cardiac magnetic resonance consistent with constrictive pericarditis. 
pericarditis ( $\mathrm{n}=16 / 37$ in this study); however, it may be true that vast majority of patients with constrictive pericarditis have pericardial adhesions detectable with CMR (16/16 in this study). Thus using CMR to detect pericardial adhesion may be a sensitive but nonspecific means of diagnosing constrictive pericarditis. Nonetheless, CMR has the potential to obtain better specificity while remaining noninvasive by combining pericardial adhesion with other parameters, such as septal thickening, septal bounce, dilated vena cavae, interventricular dependence, and altered respiratory variation of mitral and tricuspid inflow patterns.

CMR may well offer a noninvasive means of diagnosing constrictive pericarditis from a combination of (1) absent slippage between the epicardium and the pericardium and
(2) constrictive cardiac mechanics. If this proves true, the tricky diagnosis of constriction could be much simpler and less invasive. There remain some sticking points, however, in validating this technique in a broader population more typical of that scene in Western patients with constrictive pericarditis. Once these issues have been addressed, CMR may well have broad applicability in avoiding some of the invasiveness and learning curve associated with diagnosing constrictive pericarditis by right heart catheterization.

\section{Reference}

1. Power JA, Thompson DV, Rayarao G, Doyle M, Biederman RW. Cardiac magnetic resonance radiofrequency tissue tagging for diagnosis of constrictive pericarditis: a proof of concept study. J Thorac Cardiovasc Surg. 2016;151: $1348-55$. 